\title{
Newborn screening for homocystinurias and methylation disorders: systematic review and proposed guidelines
}

\author{
Martina Huemer • Viktor Kožich • Piero Rinaldo • \\ Matthias R. Baumgartner • Begoña Merinero • \\ Elisabetta Pasquini • Antonia Ribes • Henk J. Blom
}

Received: 12 January 2015 / Revised: 12 February 2015 / Accepted: 23 February 2015 / Published online: 12 March 2015

(C) SSIEM 2015

\begin{abstract}
Newborn screening (NBS) is justified if early intervention is effective in a disorder generally not detected early in life on a clinical basis, and if sensitive and specific biochemical markers exist. Experience with NBS for homocystinurias and methylation disorders is limited. However, there is robust evidence for the success of early treatment with diet, betaine and/or pyridoxine for CBS deficiency and good evidence for the success of early betaine treatment in severe MTHFR deficiency. These conditions can be screened in dried blood spots by determining methionine (Met), methionine-to-phenylanine (Met/Phe) ratio, and total homocysteine (tHcy) as a second tier marker. Therefore, we recommend NBS for cystathionine beta-synthase and severe MTHFR deficiency. Weaker evidence is available for the disorders of intracellular cobalamin
\end{abstract}

Communicated by Bridget Wilcken

Martina Huemer and Viktor Kožich contributed equally to this work.

M. Huemer · M. R. Baumgartner

Division of Metabolism and Children's Research Center,

University Children's Hospital Zürich, Zürich, Switzerland

M. Huemer · M. R. Baumgartner

Radiz-Rare Disease Initiative Zürich, University Zürich,

Zürich, Switzerland

M. Huemer $(\square)$

Department of Pediatrics, Landeskrankenhaus Bregenz, Carl-Pedenz-Str. 2, 6900 Bregenz, Austria

e-mail: martina.huemer@lkhb.at

\section{Kožich}

Institute of Inherited Metabolic Disorders, Charles University in Prague-1st Faculty of Medicine and General University Hospital,

Prague, Czech Republic

P. Rinaldo

Department Laboratory Medicine and Pathology, Mayo Clinic,

Rochester, MN, USA metabolism. Early treatment is clearly of advantage for patients with the late-onset cblC defect. In the early-onset type, survival and non-neurological symptoms improve but the effect on neurocognitive development is uncertain. The cblC defect can be screened by measuring propionylcarnitine, propionylcarnitine-to-acetylcarnitine ratio combined with the second tier markers methylmalonic acid and tHcy. For the $\mathrm{cblE}$ and cblG defects, evidence for the benefit of early treatment is weaker; and data on performance of Met, Met/Phe and tHcy even more limited. Individuals homozygous or compound heterozygous for MATIA mutations may benefit from detection by NBS using Met, which on the other hand also detects asymptomatic heterozygotes. Clinical and laboratory data is insufficient to develop any recommendation on NBS

B. Merinero

Centro de Diagnóstico de Enfermedades Moleculares, Facultad de Ciencias, Universidad Autónoma de Madrid, IDIPAZ, CIBER de Enfermedades Raras, Madrid, Spain

E. Pasquini

Department of Neuroscience, Newborn Screening Unit, A. Meyer University Children's Hospital, Florence, Italy

A. Ribes

Division Inborn Errors of Metabolism, Hospital Clinic, CIBERER, Barcelona, Spain

H. J. Blom

Laboratory Clinical Biochemistry and Metabolism, Center for Pediatrics and Adolescent Medicine University Hospital, Freiburg, Freiburg, Germany 
for the cblD, cblF, cblJ defects, glycineN-methyltransferase-, S-adenosylhomocysteinehydrolase- and adenosine kinase deficiency.

$\begin{array}{ll}\text { Abbreviations } \\ \text { ADK } & \text { adenosine kinase } \\ \text { C2 } & \text { carnitine, acetylcarnitine } \\ \text { C3 } & \text { carnitine propionylcarnitine } \\ \text { CBS } & \text { cystathionine beta-synthase } \\ \text { DBS } & \text { dried blood spots } \\ \text { GNMT } & \text { glycine-N-methyltransferase } \\ \text { Hcy } & \text { homocysteine } \\ \text { MAT } & \text { methionine adenosyltransferase } \\ \text { MMA } & \text { methylmalonic acid } \\ \text { Met } & \text { methionine } \\ \text { MTHFR } & \text { methylenetetrahydrofolate reductase } \\ \text { NBS } & \text { newborn screening } \\ \text { OH-Cbl } & \text { hydroxycobalamin } \\ \text { R4S } & \text { Region 4 Stork } \\ \text { SAHH } & \text { S-adenosylhomocysteine hydrolase } \\ \text { tHcy } & \text { total homocysteine }\end{array}$

\section{Introduction}

One of the major problems in the evaluation of data on clinical course and treatment efficacy in rare diseases is the absence of standardized databases derived from large numbers of patients assembled in an unbiased manner. Such data on natural course and benefits of early intervention are essential to support decisions on what to include in newborn screening (NBS) panels. To meet these needs for a specific group of inborn errors of metabolism, E-HOD, the European network and registry for homocystinurias and methylation defects (disturbed S-adenosylmethionine and S-adenosylhomocysteine ratio) was started in February 2013 (Table 1, pathways depicted in Fig. 1). Aims of the project are, among others, the establishment of a disease registry for the collection of clinical, biochemical and genetic data, and the development of guidelines.

The aims above are pursued in this report with respect to guidelines for NBS for the homocystinurias and methylation disorders developed on the basis of a systematic review of the literature, current NBS practice and expert discussions.

\section{Methods}

A systematic search of the Pubmed and Cochrane databases was performed using the search term "newbornscreening" in combination with every disease addressed by this report. To evaluate treatment efficacy, publications on clinical course, outcome and treatment were searched for.

After exclusion of publications containing no relevant information, study design and level of evidence was evaluated for 103 publications according to the "Grading of Recommendations Assessment, Development and Evaluation (GRADE) working group (http://www.gradeworkinggroup.org/)" approach. Furthermore, data on metabolite concentrations in dried blood spots (DBS) at the time of screening obtained from 486 patients with subsequently confirmed homocystinurias and methylation disorders as well as cumulative data on distribution of analytes in healthy newborns and clinically validated target ranges for cut-off values were retrieved from the multi-center study "Region 4 Stork" (R4S) website (McHugh et al 2011). In addition, EHOD partners were asked to contribute their non-published experience with NBS for the disorders of interest. The guidelines were established based on the literature evaluation and expert discussions following the GRADE approach. Expert consensus was targeted for each disease on the following key questions:

a) Is the natural course of the disease severe?

b) Is treatment generally beneficial?

c) Is early intervention more effective?

d) Are robust, valid and reliable methods, screening approaches and strategies available?

\section{Results}

Classical homocystinuria or cystathionine- $\beta$-synthase deficiency

\section{Clinical findings}

In classical homocystinuria or cystathionine- $\beta$-synthase [CBS] deficiency, the condensation of serine and homocysteine (Hcy) to form cystathionine is disturbed as well as other processes such as the production of hydrogen sulphide from other substrates. Pyridoxal 5'-phosphate formed from dietary vitamin $B_{6}$ is the essential cofactor of the enzyme. The true incidence of CBS deficiency is unknown; it has been estimated at 1:344.000 worldwide (Mudd et al 1985) but due to different population frequencies and ascertainment methods, reports on CBS incidence vary largely. Molecular epidemiological studies predict frequencies between 1:6.400 and 1:20.500 in European populations (Gaustadnes et al 1999; Refsum et al 2004; Janosík et al 2009).

Biochemical hallmarks of CBS deficiency are highly elevated concentrations of plasma total homocysteine (tHcy) combined with low plasma cystine or total cysteine, high normal to elevated plasma methionine (Met), low normal to 


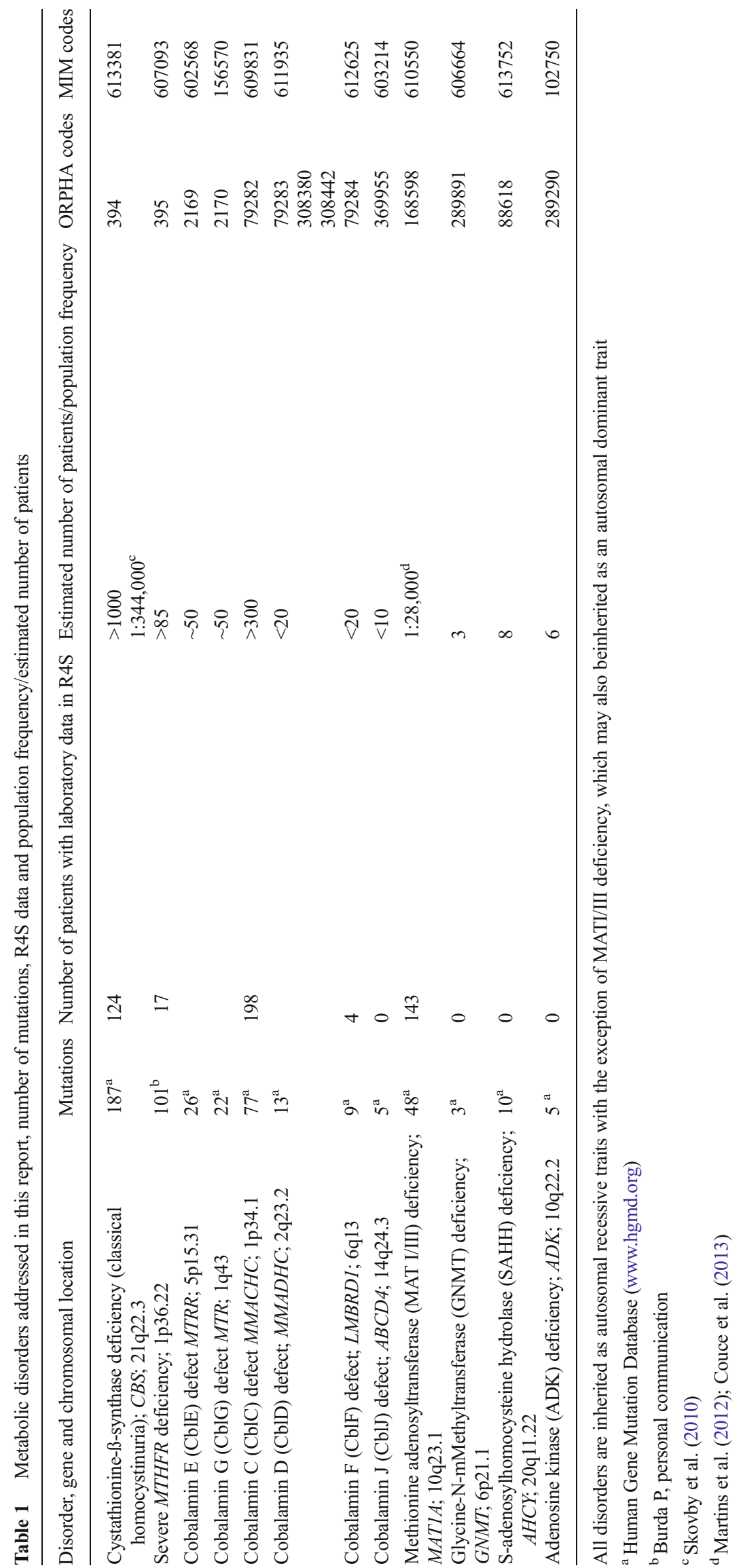




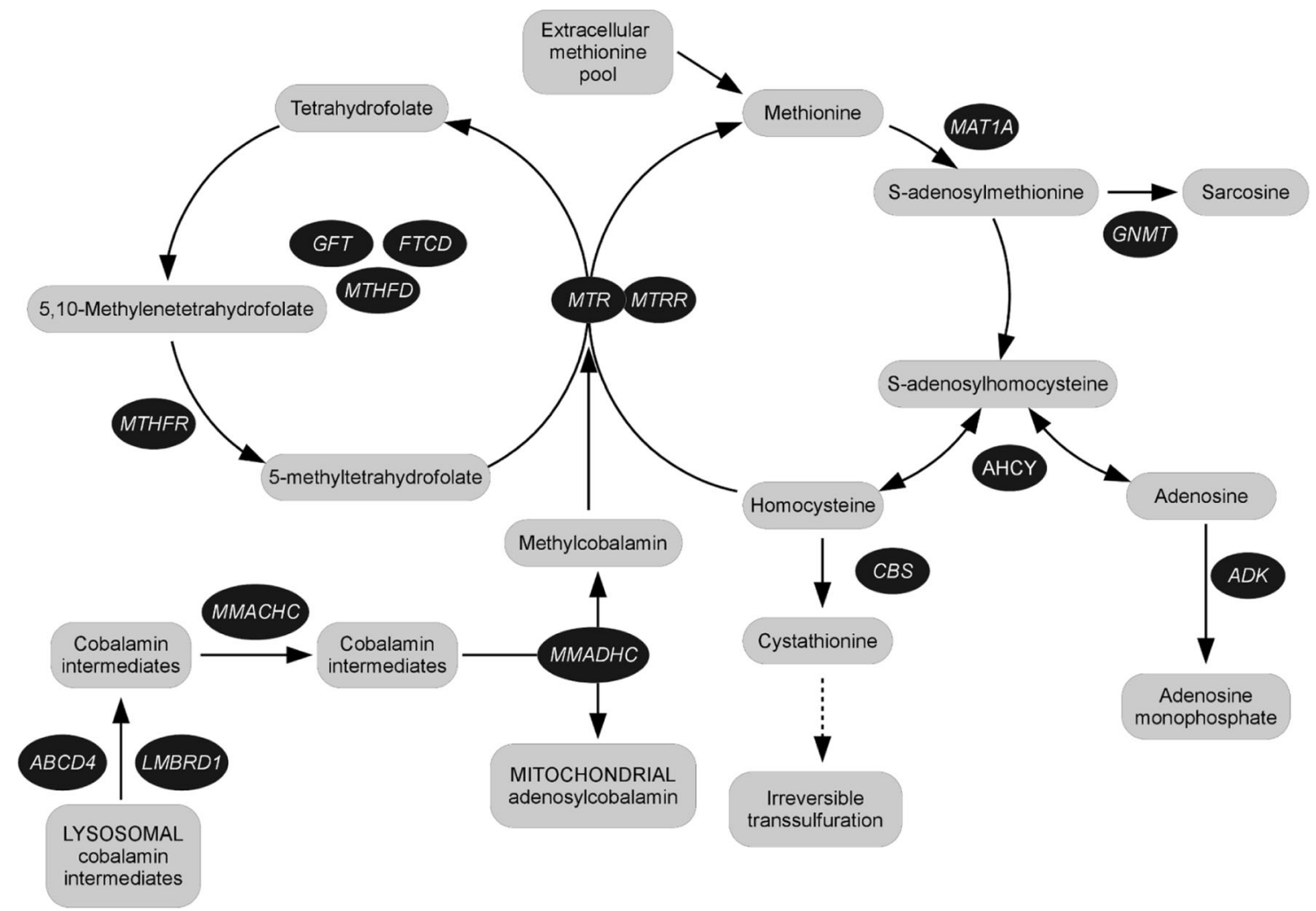

Fig. 1 Homocystinurias and methylation disorders: metabolic pathways

decreased cystathionine, and a grossly abnormal cystathionine/Met ratio (Stabler et al 2013; Bártl et al 2014). Generally, patients present with ectopia lentis and myopia, mental retardation, marfanoid habitus and osteoporosis, thromboembolic events, developmental and behavioral problems (Mudd et al 1985). If diagnosis is delayed, significant long-term morbidity must be expected (Naughten et al 1998; Yap and Naughten 1998). Milder forms and especially the pyridoxine-responsive variants of the disease due to specific mutations such as c.833 $\mathrm{T}>\mathrm{C}$ (p.I278T) may manifest later in adulthood e.g., with thromboembolic events or may even remain asymptomatic until adulthood (Skovby et al 2010; Magner et al 2011).

In the pyridoxine-responsive patients, variable doses of pyridoxine elicit near-normalization of biochemical parameters (Mudd et al 1985). Patients with the more severe, pyridoxine non-responsive form of the disease are generally treated with a Met restricted diet mostly combined with supplementing amino acid mixtures enriched with cysteine. Many patients are treated with oral betaine, which provides methyl groups for homocysteine [Hcy] remethylation to Met (Adam et al 2013). Treatment encompassed a combination of diet and betaine in $61 \%$, betaine alone in $34 \%$ and diet alone in $5 \%$ of 181 European pyridoxine non-responsive CBS patients (Adam et al 2013).

Despite the variability of approaches, treatment effectively reduces morbidity, especially when initiated early. A significant reduction of vascular morbidity has been observed in 158 treated patients (Yap et al 2001). While 112 vascular complications were predicted on the basis of historical data from untreated CBS deficient patients, only 17 vascular complications occurred in 12 patients (Yap et al 2001). Thirteen CBS patients identified by NBS and with good compliance to treatment had normal full-scale IQ results (mean 105.8) in contrast to six poorly compliant (mean IQ 80.8) and two untreated patients (IQ 52 and 53) (Yap et al 2001). These findings are supported by 11 patients diagnosed on NBS who developed no somatic symptoms and had a normal IQ (Walter et al 1998). In 14 late diagnosed patients, all had lens subluxation/dislocation and impaired vision at diagnosis and eye disease progressed with less strict adherence to treatment. In contrast, 15 early treated patients had no evidence of lens subluxation and better visual acuity (Mulvihill et al 2001).

Recommendation The severe form of CBS deficiency is defined by its significant morbidity (milder forms are also known). A large subgroup of patients is responsive to pyridoxine administration. Low methionine/low protein diet (mostly combined with methionine-free and cysteine enriched amino acid mixture) and/or betaine administration is effective in pyridoxine non-responsive CBS deficiency. Normal cognitive development and prevention of physical 
complications is possible in patients detected by NBS and treated early with continuous strict compliance.

\section{Methods for NBS in CBS deficiency}

Since the 1970s NBS for CBS deficiency has been based on the detection of increased Met concentration in dried blood spots (DBS) (Chace et al 1996; Mudd 2011). Long-term experience with the NBS for CBS deficiency exists namely in Ireland, Italy, Portugal, and some U.S. states (McHugh et al 2011; Naughten et al 1998; Peterschmitt et al 1999; Accinni et al 2003; Martins et al 2012). Varying proportions (20-50\%) of missed pyridoxine non-responsive cases have been reported with different Met cut-off values used (Naughten et al 1998; Gan-Schreier et al 2010). Lowering of the Met cut-off from 138 to $67 \mu \mathrm{mol} / \mathrm{L}$ doubled the detection rate of CBS deficiency (Peterschmitt et al 1999). Accordingly, Met cutoff values as low as $40 \mu \mathrm{mol} / \mathrm{L}$ have been proposed to yield higher sensitivity (McHugh et al 2011; Turgeon et al 2010). Sensitivity is also increased by using the Met/Phe ratio which adjusts for protein intake (Bowron et al 2005). In conclusion, the primary factor driving sensitivity is the selection of as low as possible cut-off values for Met and Met/Phe ratio.

Although the pyridoxine responsive form of CBS deficiency is common in various populations and represented $44 \%$ of patients in the historical cohort of 629 patients (Mudd et al 1985), only 3-13\% of patients detected by NBS reported in the literature were pyridoxine responders (Mudd et al 1985; Janosík et al 2009). This marked discrepancy indicates that NBS programs using Met as the primary marker most likely fail to detect the majority of patients with pyridoxine responsive disease (Mudd 2011).

Specificity of Met as the primary marker is poor since blood concentrations also increase in other disorders, e.g., liver disease, MATI/III, GNMT and SAHH deficiencies, and tyrosinemia type I (Mudd 2011). Specificity may be improved by quantifying tHcy in DBS as a second-tier marker (Bártl et al 2014; Turgeon et al 2010; Alodaib et al 2012) leading to a fivefold reduction of the false positive rate compared to the period when only Met at a cut-off of $40 \mu \mathrm{mol} / \mathrm{L}$ was used (Bowron et al 2005).

Data on 124 CBS deficient patients ascertained by NBS show wide variation of the Met concentration in DBS with a clearly increased median of $103 \mu \mathrm{mol} / \mathrm{L}$ compared to the median of $20 \mu \mathrm{mol} / \mathrm{L}$ in healthy neonates (Table 3) (McHugh et al 2011). A target cut-off value for Met to differentiate the 99th centile in healthy newborns from the 5th centile in confirmed true positive cases recommended by R4S is in the range between 39 and $50 \mu \mathrm{mol} / \mathrm{L}$. The Met/Phe ratio has slightly better performance characteristics. Data on tHcy in DBS as a secondary marker at the time of screening is available from 31 CBS deficient patients showing a clear distinction between cases (median tHcy $40 \mu \mathrm{mol} / \mathrm{L}$ ) and controls
$(6 \mu \mathrm{mol} / \mathrm{L})$. When tHcy is measured on the same specimen as a second tier test, calculating the Met/tHcy ratio may help differentiate the patients with CBS deficiency from MATI/III deficient individuals (see Table 3).

The measurement of tHcy as primary NBS marker has only been used in the native Qatari population due to technical requirements and high costs (Gan-Schreier et al 2010). Molecular genetic testing is feasible and may be an option for high-risk populations with a limited number of prevalent mutations. However, this approach will miss individuals carrying other mutations (Gan-Schreier et al 2010).

Recommendation NBS for CBS deficiency can be performed by detecting elevated methionine, methionine-tophenylalanine ratio and/or hyperhomocysteinemia in dried blood spots. However, total homocysteine has only exceptionally been used as a primary marker. Sensitivity of methionine as a primary marker for pyridoxine non-responsive CBS deficiency is limited and inversely related to the chosen cut-off concentrations of methionine. For the pyridoxine responsive form of the disease sensitivity is largely unknown and probably low. Specificity of methionine as a primary marker may be substantially increased by analyzing total homocysteine as a second tier marker and calculating the Met/tHcy ratio.

The combined remethylation defects cblC, cblF, cblJ, and cblD-MMA-Hcy

\section{Clinical findings}

The cblC defect, caused by mutations in the MMACHC gene is the most common of these defects (Lerner-Ellis et al 2009). Mutations in the $L M B R D 1$ gene cause the cblF defect (Rutsch et al 2009), and $A B C D 4$ mutations the cblJ defect (Coelho et al 2012). Depending on type and location of the mutation in the MMADHC gene, the cblD defect either presents as isolated methylmalonic acid (MMA)-uria (cblD-MMA, beyond the scope of this report), combined MMA and homocystinuria (cblD-MMA-Hcy, resembling the cblC, cblF and cblJ defects) or isolated homocystinuria (cblD-Hcy, clinically indistinguishable from the cblE or cblG defect, addressed below) (Jusufi et al 2014). All defects affect the synthesis of methylcobalamin, the co-factor for the cytosolic enzyme methionine synthase, and of adenosylcobalamin, the coenzyme for the intramitochondrial enzyme methylmalonyl-CoA mutase, which metabolizes MMA (Fig. 1). Thus low Met, elevated tHcy, propionylcarnitine and MMA are biochemical hallmarks of the disorders (McHugh et al 2011; Fowler 1998).

Main clinical symptoms of the early-onset form of the cblC defect ( $<12$ months, $88 \%$ of cases) (Fischer et al 2014) include failure to thrive, macrocytic anemia, muscular hypotonia, developmental delay, dysmorphic features, thinned corpus callosum, hydrocephalus, hemolytic uremic syndrome, 
and decreased visual acuity due to pigmentary retinopathy but no lens dislocation (Rosenblatt et al 1997; Weisfeld-Adams et al 2013; Fischer et al 2014). Late-onset patients may present any time during childhood or adult life, typically with various combinations of myelopathy, dementia, psychiatric symptoms, hemolytic-uremic syndrome, thromboembolism, and pulmonary hypertension; retinopathy occurs rarely (Fischer et al 2014; Huemer et al 2014a).

Treatment of the cblC defect - which has also been applied in cblF, cblJ, and cblD-Hcy-MMA defects - consists of intramuscular hydroxycobalamin $(\mathrm{OH}-\mathrm{Cbl})$, oral betaine and folate, rarely combined with Met supplementation. Carnitine is often added but has no proven effect (Martinelli et al 2011). Dosages of medications vary considerably (Fischer et al 2014; Carrillo-Carrasco et al 2012).

In early-onset cblC patients, overall survival, hematological symptoms, and failure to thrive improve while eye disease and cognitive impairment often progress under treatment (Martinelli et al 2011; Fischer et al 2014). Despite identification by NBS and early treatment, 11/12 patients showed developmental delay, 8/9 brain pathologies, 10/11 muscular hypotonia, and $9 / 12$ nystagmus at a mean age of 50 months (Weisfeld-Adams et al 2013). However, from a screened and early treated cohort of 13 patients from Tuscany, the ten patients subjected to psychometric testing achieved a mean DQ of 80 or IQ of 85 compared to a mean DQ/IQ of 45 and 66 in 18 clinically diagnosed patients (mean age at diagnosis: 54 days). Only 7/13 screened and early treated patients developed the characteristic eye disease compared to $11 / 13$ clinically diagnosed early-onset patients (Pasquini 2014, personal communication).

In most late-onset cblC patients, dementia, renal function, myelopathy, and axonal neuropathy improve on treatment. Long-lasting disease before treatment initiation correlates with incomplete recovery and residual pathologies (Huemer et al 2014a).

Clinical information is available for less than twenty patients with the cblF defect. Three patients died at 5, 9, and 10 months, respectively (Shih et al 1989; Bowron et al 2005; Alfadhel et al 2011). In some early treated patients, neurocognitive outcome was good (Miousse et al 2011; Armour et al 2013), while in others severe developmental delay was present (Gailus et al 2010; Alfadhel et al 2011). For the cblJ defect (Coelho et al 2012), clinical information on treatment outcome is extremely limited. Two patients reportedly responded well to treatment with methylcobalamin (Kim et al 2012). In the reported cblD-Hcy-MMA cases, a broad spectrum of responses to treatment has been observed ranging from fatal progression of symptoms (Miousse et al 2009) to improved (Suormala et al 2004; Miousse et al 2009) to even nearly normal development (Suormala et al 2004) (Table 2).
Recommendation Screening for the cblC defect should be considered since in the early-onset $\mathrm{CblC}$ defect response to treatment has been observed for non-neurological symptoms with improved overall survival. The impact of early treatment on neurocognitive development and retinopathy is unclear. For late-onset patients treatment is mostly beneficial, especially when initiated before irreversible organ damage has occurred. The present knowledge allows no conclusion concerning the benefit of early treatment for the cblD-HcyMMA, cblF and cblJ defects.

\section{Methods for NBS in the combined remethylation defects}

Newborn screening for these defects has used the primary markers propionylcarnitine (C3), propionylcarnitine/ acetylcarnitine $(\mathrm{C} 3 / \mathrm{C} 2)$ ratio, Met, and Met/Phe ratio. The $\mathrm{R} 4 \mathrm{~S}$ data indicate that the sensitivity of $\mathrm{C} 3 / \mathrm{C} 2$ is superior to Met and Met/Phe. Indeed, the use of cut-off values for $\mathrm{C} 3 / \mathrm{C} 2$ between 0.18 and $0.20 \mu \mathrm{mol} / \mathrm{L}$ differentiates between the 99 th centile in healthy newborns and the 5 th centile of cases with cblC, cblD-Hcy-MMA and cblF (McHugh et al 2011). In contrast, both Met and Met/Phe will miss a considerable proportion of cases if used as a primary marker alone (McHugh et al 2011) (Table 3).

The specificity of $\mathrm{C} 3$ and/or $\mathrm{C} 3 / \mathrm{C} 2$ for detecting these diseases is generally low (la Marca et al 2007; Turgeon et al 2010) since they also detect nutritional imbalances (including maternal vitamin B12 deficiency) or other inborn errors of metabolism (Scolamiero et al 2014). Low Met has been proposed as a possible secondary marker (Weisfeld-Adams et al 2010), but due to the above mentioned shortcomings, other second tier strategies have been developed, applying isolated or simultaneous measurement of MMA, methylcitric acid and tHcy (Alodaib et al 2012; la Marca et al 2007; Tortorelli et al 2010; Fu et al 2013). While methylcitric acid does not differentiate $\mathrm{cblC} / \mathrm{cblD}$ patients from controls, the positive predictive value substantially increased from 4 to $100 \%$ by measurement of MMA and from 11 to $36 \%$ by measuring tHcy (la Marca et al 2007; Turgeon et al 2010).

Recommendation NBS for the cblC defect can be performed by identification of elevated propionylcarnitine with a high propionylcarnitine/acetylcarnitine ratio. Low methionine or low methionine/phenylalanine ratio are less sensitive. Sensitivity of propionylcarnitine and propionylcarnitine/ acetylcarnitine ratio for detecting mild forms of the cblC defect is unknown. Specificity of biomarkers substantially increases by analyzing methylmalonic acid and/or total homocysteine in DBS as a second tier test. It can be anticipated that the same markers are useful for the detection of cblD-HcyMMA, cblF or cblJ disorders but efficacy and feasibility of screening for these three disorders is unknown. It should be 
Table 2 Disease course and treatment response in homocystinurias and methylation disorders

\begin{tabular}{|c|c|c|}
\hline Disease & Disease course & Response to treatment \\
\hline CBS deficiency $\mathrm{B}_{6}$-non responsive & Severe & $\begin{array}{l}\text { Early, strict treatment results in favorable physical and } \\
\text { cognitive outcome. }\end{array}$ \\
\hline CBS deficiency $\mathrm{B}_{6}$-responsive & Mild to asymptomatic & Good response to pyridoxine \\
\hline cblC defect & Severe & $\begin{array}{l}\text { Early-onset: } \\
\text { Survival, hematological and microangiopathy } \\
\text { symptoms responsive. } \\
\text { Neurological, opthalmological and developmental } \\
\text { problems often ongoing } \\
\text { Late-onset: } \\
\text { Predominantly responsive, residual myelopathy/neuropathy } \\
\text { frequent in prolonged untreated courses }\end{array}$ \\
\hline $\begin{array}{l}\text { cblD-Hcy defect } \\
\text { cblD-MMA-Hcy defect }\end{array}$ & Severe & Variable, very limited data \\
\hline cblF defect & Severe & Variable, very limited data \\
\hline cblJ defect & Severe & Variable, very limited data \\
\hline Severe MTHFR deficiency & Severe & Early betaine treatment is beneficial \\
\hline cblE and cblG defect & Severe & $\begin{array}{l}\text { Treatment seems beneficial in a majority of patients } \\
\text { but response is variable. Individual case reports } \\
\text { encourage early treatment }\end{array}$ \\
\hline MAT I/III deficiency, heterozygous individuals & Predominantly benign & Generally good without treatment \\
\hline $\begin{array}{l}\text { MAT I/III deficiency, compound heterozygote } \\
\text { and homozygote individuals }\end{array}$ & $\begin{array}{l}\text { White matter disease, cognitive } \\
\text { impairment observed }\end{array}$ & $\begin{array}{l}\text { Methionine-lowering treatment may be beneficial } \\
\text { but data is very limited }\end{array}$ \\
\hline GNMT deficiency & Predominantly benign & Generally good without treatment \\
\hline SAHH deficiency & Severe & Variable, very limited data \\
\hline ADK deficiency & Severe & Unknown \\
\hline
\end{tabular}

noted that by using these markers, maternal vitamin B12 deficiency might be detected.

Isolated remethylation defects: the cblE, cblG, and cblD-Hcy defects, and severe MTHFR deficiency

\section{Clinical findings}

The cblG defect affects the enzyme methionine synthase (MTR) while methionine synthase reductase (MTRR) is defective in the cblE defect (Watkins and Rosenblatt 2012). The clinically similar cblD-Hcy defect was discussed above. All defects impair the remethylation of Hcy to Met and result in elevated tHcy and low normal to low Met (Stabler et al 2013; Huemer et al 2014b).

The clinical pattern of the cblE and the cblG defect is indistinguishable. Mostly, initial symptoms evolve in the first year of life or in early childhood (Zavadakova et al 2005). Macrocytic and/or megaloblastic anemia, failure to thrive and feeding difficulties, cognitive dysfunction, nystagmus, impaired visual acuity but no lens dislocation, and seizures are frequent. Microangiopathy and hemolytic uremic syndrome have only very rarely been described (Huemer et al 2014b). A subgroup of three patients from the Iberian Peninsula presented with isolated macrocytic anemia (Vilaseca et al 2003).
Treatment encompasses folic or folinic acid, betaine and a variety of cobalamin (OH-Cbl, cyano- or methylcobalamin) preparations. Mostly tHcy and Met as well as macrocytic anemia (Fowler et al 1997; Vilaseca et al 2003) and neurocognitive performance (Fowler et al 1997; Harding et al 1997; Vilaseca et al 2003; Müller et al 2007; Schiff et al 2011) respond well to treatment. In four patients, a positive impact of early treatment on outcome has been observed (Müller et al 2007; Schiff et al 2011). However, in a series of nine cblE patients, the overall impact of treatment on neurodevelopmental disabilities and eye disease was "at most moderate" (Zavadakova et al 2005). Eye disease seems not to respond to treatment (Huemer et al 2014b).

Severe methylenetetrahydrofolate reductase (MTHFR) deficiency also results in defective methylation of Hcy to Met. The enzyme MTHFR catalyzes the reduction of 5,10 methylenetetrahydrofolate to 5-methyltetrahydrofolate, which serves as a methyl donor for the methylation of Hcy to Met (Fattal-Valevski et al 2000). In addition to elevated tHcy and decreased Met the folate concentrations in plasma and especially in CSF are decreased in severe MTHFR deficiency (Thomas and Rosenblatt 2005; Stabler et al 2013). As in the combined defects, due to the enhanced flux through the transsulfuration pathway, plasma cystathionine (median $1192 \mathrm{nmol} / \mathrm{L}$ ) is grossly elevated in patients compared to controls (median $157 \mathrm{nmol} / \mathrm{L}$ ) (Stabler et al 2013). 


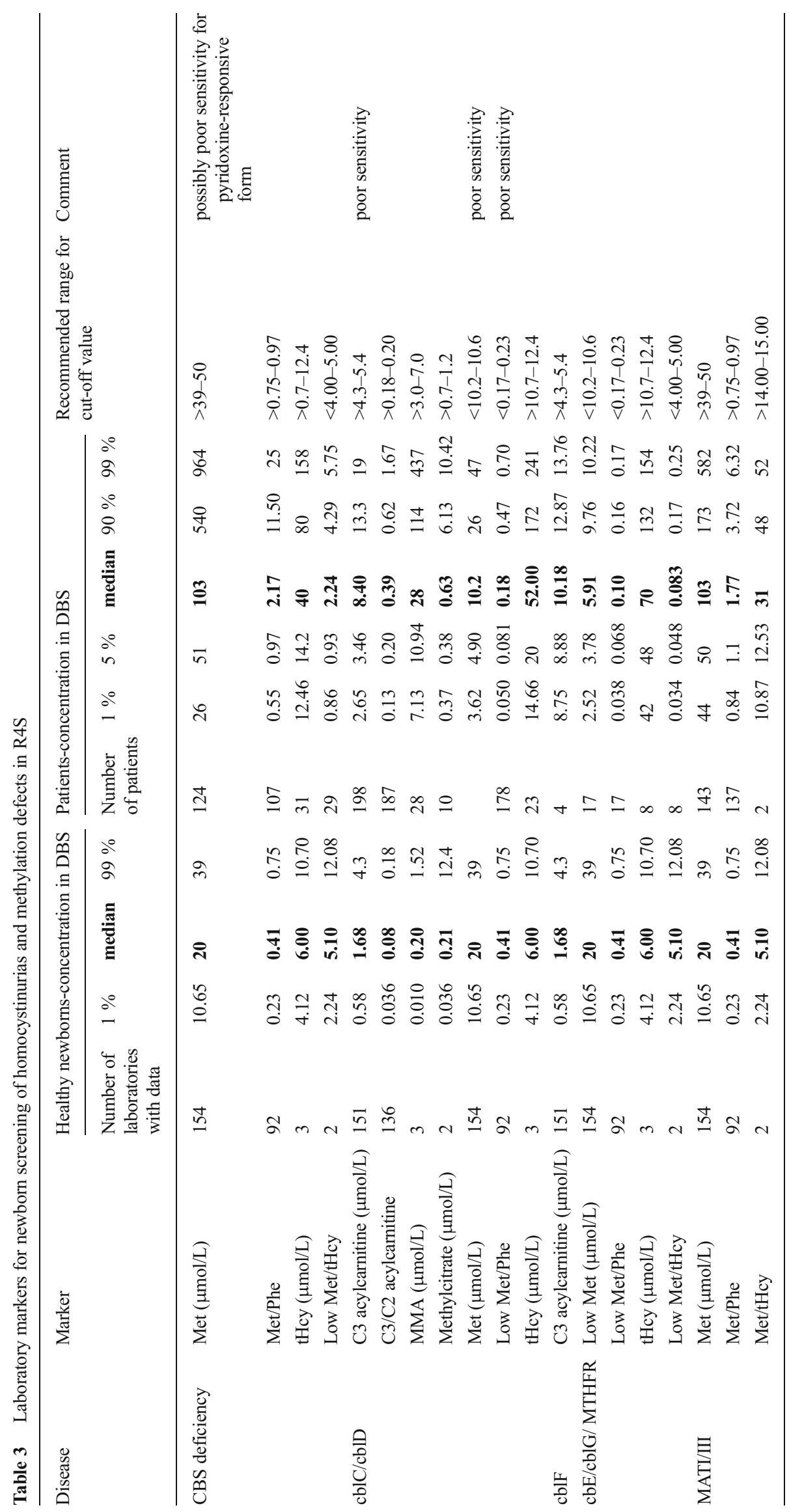


In severe MTHFR deficiency, developmental delay, seizures, and movement disorders are frequent in children. Adolescents and adults may present with psychiatric symptoms, neuropathy or thromboembolic events. Retinopathy has only rarely been reported (Thomas and Rosenblatt 2005) but data from a mouse model show impaired retinal function (Lawrance et al 2011). Megaloblastic and/or macrocytic anemia is usually absent (Thomas and Rosenblatt 2005). Treatment with folate, folinic acid, Met, pyridoxine, and different cobalamin preparations has generally been considered unsuccessful (Thomas and Rosenblatt 2005). However, Met supplementation improved the course in an early onset case (Abeling et al 1999). In a systematic review including 36 patients, the positive impact of early betaine treatment on outcome in earlyonset patients was shown (Diekman et al 2014). Five early treated patients survived with normal psychomotor development while nine of ten non-treated and two late treated patients died. In families with one or more deceased siblings, none of the treated but all untreated children died. Psychomotor development was impaired in all children with delayed treatment onset, despite stabilization from introduction of treatment (Diekman et al 2014) (Table 2).

Recommendation NBS for severe MTHFR deficiency is recommended since early betaine administration is associated with a positive outcome. NBS for the cblD-Hcy, cblE, and cblG defects can be considered since in the majority of clinically diagnosed patients, treatment seems beneficial and there is some evidence for an added benefit of early treatment.

\section{Methods for NBS in the cblD-Hcy, cblE, and cblG defects, and severe MTHFR deficiency}

Neonatal screening for these disorders became possible only after the introduction of tandem mass spectrometry for determination of decreased Met concentration in DBS (Chace et al 1996). Data on 18 remethylation cases were available in R4S (four MTHFR, one cblG, and one cblE and 12 non-classified cases). The Met concentration in the DBS was decreased to a median of $5.91 \mu \mathrm{mol} / \mathrm{L}$ in the patients, with no overlap with healthy newborns (Table 3). In addition, both the Met/Phe ratio (Bowron et al 2005) and the second-tier marker tHcy clearly differentiate patients from controls (Tortorelli et al 2010; McHugh et al 2011).

In theory, tHcy can be employed as the primary screening marker but there are no published data on its use. There are no long-term data on efficacy of screening programs for these disorders (Tortorelli et al 2010) owing to their rarity and low proportion of screening programs addressing decreased Met or Met/Phe ratio (in R4S, 162 laboratories have listed a high cut-off for methionine, but only 73 have a low cut-off value).
Recommendation Neonatal screening for the cblD-Hcy, cblE, and cblG defects, and for MTHFR deficiency appears to be possible by detecting a decreased methionine and methionine-to-phenylalanine ratio in DBS followed by analysis of total homocysteine as a second tier marker. Efficacy and feasibility of screening for these disorders is largely unknown.

Methionine adenosyltransferase deficiency

\section{Clinical findings}

Methionine adenosyltransferase (MAT) catalyses the formation of S-adenosylmethionine (AdoMet) from Met. Three variant forms (MAT I, II and III) of the enzyme have been described. The MAT I and III - encoded by a single gene $(M A T 1 A)$ - are expressed mainly in liver. The MATII is expressed ubiquitously in extrahepatic tissues and seems to play a pivotal role in maintaining whole body AdoMet homeostasis. Homozygosity or compound heterozygosity for MAT1A mutations and heterozygosity for some mutations result in hypermethioninemia without elevation of AdoMet concentrations. Inheritance of this biochemical trait may thus be autosomal recessive or autosomal dominant (Baric 2009).

There is evidence that a small number of individuals homozygous or compound heterozygous for MATIA mutations develop myelination defects or white matter disease and some of these patients are cognitively impaired. These clinical signs have been related to persistent very high Met concentrations and/or to AdoMet deficiency in the brain $(17,63)$. It is advisable to monitor these patients closely and to consider introduction of a Met restricted diet or AdoMet supplementation. The available evidence suggests that AdoMet supplementation may normalize existing white matter changes (Surtees et al. 1991).

However, the most common autosomal dominant form of hypermethioninemia detected by neonatal screening is caused by heterozygosity for the mutation c. 791G > A (p.R264H). Since the clinical course in these individuals is generally benign (Baric 2009; Mudd 2011), the impact of a proteincontrolled diet cannot clearly be evaluated (Couce et al 2013) and the usefulness of early detection of these individuals is questionable (Mudd 2011; Blom 2014, personal communication) (Table 2).

Recommendation In individuals heterozygous for MAT I/ III deficiency, treatment seems not to be necessary. Patients homozygous/compound heterozygous for MAT I/ III mutations should be monitored closely and may require intervention. Data on the benefit of early intervention is lacking. 


\section{Methods for NBS in MATI/III}

Most MAT I/III deficient individuals were originally identified by NBS programs targeting CBS deficiency (Mudd 2011) and the number of detected individuals substantially increased with use of lower Met cut-off values between 45 and $54 \mu \mathrm{mol} /$ $\mathrm{L}$ (Martins et al 2012). The incidence is especially high in the Galicia region of Spain and in Portugal (1:26.000-1: 28.163), mostly due to heterozygosity for the p.R264H mutation (Martins et al 2012; Couce et al 2013). The DBS concentrations of Met in $143 \mathrm{MAT}$ I/III deficient individuals (including carriers) in the R4S database reached a median of $103 \mu \mathrm{mol} / \mathrm{L}$ compared to the median of $20 \mu \mathrm{mol} / \mathrm{L}$ in healthy neonates (McHugh et al 2011). A target cut-off value for Met to differentiate between the 99th centile in healthy newborns from the 5th centile in confirmed true positive cases is consequently in the range between 39 and $50 \mu \mathrm{mol} / \mathrm{L}$ (Table 3). The Met/Phe ratio has similar performance characteristics (McHugh et al 2011). Differentiation between MATI/III and CBS deficiency based on Met concentrations is not possible. However, determination of tHcy concentration as second tier strategy and then calculating the Met/tHcy ratio may discriminate between CBS and MATI/III deficiency; a simple and effective approach is to apply the dual scatter plot functionality available on the website of the R4S collaborative project (Table 3).

Recommendation Individuals with MATI/III deficiency have been predominantly detected in NBS programs for CBS deficiency due to increased methionine concentrations. The majority of these newborns are heterozygotes for the mutation c. $791 \mathrm{G}>\mathrm{A}$ and the benefit of detecting these individuals is questionable. At present, there is no primary marker to differentiate between MATI/III and CBS deficiency. Data on the Met/tHcy ratio to differentiate between these two conditions are promising but limited.

Glycine-N-methyltransferase deficiency,

S-adenosylhomocysteine hydrolase deficiency and adenosine kinase deficiency

Another disorder characterized by isolated hypermethioninemia is glycine-N-methyltransferase (GNMT) deficiency, which has only been described in three individuals. GNMT transfers a methyl group from Sadenosylmethionine to glycine and thus synthesizes sarcosine. Deficiency of the enzyme results in gross elevation of AdoMet and Met concentrations. GNMT deficiency seems to be a relatively benign disorder presenting with mild hepatomegaly or moderately elevated liver transaminases (Baric 2009). At present, the rarity and the benign course of the condition do not support inclusion in NBS programmes (Mudd 2011).
The enzyme S-adenosylhomocysteine hydrolase (SAHH) catalyses the hydrolysis of S-adenosylhomocysteine to Hcy and adenosine. SAHH deficiency has been described in seven patients (Honzík et al 2012). Biochemical findings include grossly increased S-adenosylhomocysteine with elevation of S-adenosylmethionine and Met while tHcy may be normal or mildly elevated. The clinical course in the first male index patient from Croatia revealed a severe disease with myelination disorder and low IQ, hepatopathy and myopathy with elevated creatine kinase. The pathogenesis of myopathy in SAHH deficiency is at present not completely understood. While Met restriction had a positive impact on neurocognitive symptoms even in the index patient and improved the course significantly in the patient's two early diagnosed and treated brothers, myopathy persisted in all cases (Barić et al 2005; Baric 2009). In another patient, treatment from the age of 5 months to 5 years failed to prevent clinical symptoms (Baric 2009; Mudd 2011). Recently two siblings with hydrops fetalis and fatal outcome have been reported (Grubbs et al 2010), underlining the severity of the disease.

The enzyme ADK catalyzes the phosphorylation of adenosine, which is produced by hydrolysis of Sadenosylhomocysteine by SAHH. When adenosine is not further metabolized, its accumulation inhibits Sadenosylhomocysteine hydrolase leading also to an increase of the upstream metabolites S-adenosylmethionine and methionine (Bjursell et al 2011). Until now adenosine kinase (ADK) deficiency has been described in six patients: two Swedish siblings and two related pairs of Malaysian siblings with severe developmental delay, macrocephaly, epilepsy, and abnormal liver function. One patient died at the age of 10 years. All patients shared the biochemical pattern of elevated methionine, S-adenosylmethionine, and Sadenosylhomocysteine levels in the presence of normal/ slightly elevated tHcy similar to SAHH deficiency. The detection of increased urinary adenosine may be useful to differentiate this defect from SAHH deficiency, in which elevated creatine kinase activity can also be expected. Methionine restriction may be beneficial in some patients (Mudd 2011). Patients may be identified by NBS programs based on elevated Met (Buist et al 2006), however no targeted NBS approaches for SAHH and ADK deficiencies have been reported so far.

Recommendation GNMT appears to be a benign condition. In the very small numbers of patients described, SAHH and ADK deficiency are predominantly severe disorders. Present knowledge allows no conclusive statement concerning the benefit of early treatment for SAHH and ADK deficiency. Neonatal screening for SAHH and ADK deficiency cannot be recommended at this stage due to lack of data and validated screening strategies. 


\section{Conclusion}

Selection of diseases for NBS must be driven by clinical arguments - a severe disease, generally not detectable early in life on a clinical basis, feasible and effective treatment, and a better outcome in early treated individuals meets accepted criteria for inclusion into a NBS program (Wilson and Jungner 1968). However, the evidence for efficacy of treatment in the homocystinurias and methylation disorders is limited due to small series of subjects with different genotypes, varying treatment, and inconsistent follow-up strategies. Particularly, time of initiation of treatment and the use, dosage, and intervals of application of drugs are far from uniform.

However, despite the above-mentioned limitations of clinical data, there is robust evidence for successful treatment and improved outcome by early treatment for CBS deficiency, and to a lesser extent for MTHFR deficiency and the cblC defect. In CBS deficiency, good outcome in individuals identified by NBS is consistently reported even from various centers including patients with a variety of $C B S$ mutations and despite a large variability in treatment practice (Naughten et al 1998; Walter et al 1998). In MTHFR deficiency, the clear clinical effect of early treatment with betaine is an important supporting evidence for screening (Diekman et al 2014). In the late-onset cblC defect, treatment response is seen in almost all patients but residual disease manifestations are frequent in patients with long duration of clinical abnormalities before treatment initiation (Huemer et al 2014a). Evidence for efficacy of early treatment is less clear for the early-onset cblC defect in which survival, hematological symptoms, and feeding problems often improve, while the effect on neurocognitive development and retinopathy is less encouraging (Weisfeld-Adams et al 2013).

If screening for these disorders is advisable from a clinical point of view, robust, applicable, and widely accepted primary and secondary markers must be used. The respective markers and ratios should be developed and their utility tested by combining a large amount of data and by application of postanalytical interpretative tools (Marquardt et al 2012; Hall et al 2014). Algorithms and cut-offs for markers based on small isolated case series have a significant risk of bias and may be misleading. However, even with the use of large sample numbers the selection of reliable cut-off values for NBS based on comparing data from patients detected by the NBS methods and values from healthy newborns is not trivial. Very little data is available on missed cases and milder forms of the screened diseases, which may manifest years or even decades later. Therefore, it must be kept in mind that NBS markers reflect the present state of the art, which is temporary and may change in the future due to technological advances and improved knowledge.

For the other disorders addressed by this review, there is either only weak evidence for a response to treatment and for the specific value of early treatment [cblE and cblG defects] (Vilaseca et al 2003; Zavadakova et al 2005), or insufficient data to evaluate treatment outcome [cblD, cblF, cblJ, GNMT, SAHH, and ADK deficiency]. It must be emphasized that NBS programs targeting CBS, MTHFR or cblC will most probably identify also subjects with other homocystinurias/ methylation disorders with uncertain clinical relevance and that biochemical algorithms to differentiate between all these disorders must be established. On one hand, this will allow a more differentiated view regarding the benefit of early detection and treatment for these disorders and facilitate genetic counselling for families with severely affected children. On the other hand, subjects requiring no treatment and follow-up may also be detected. A paradigm for this process is the MAT I/III defect for which data on clinical severity are conflicting. Heterozygosity for specific MATIA mutations is considered benign and these individuals should not but will be picked up. However, a small but significant number of individuals homozygous or compound heterozygous for MAT1A mutations may develop clinical complications and may benefit from treatment.

To improve the overall knowledge on homocystinurias and methylation disorders, studies on the natural course, treatment, and outcome following a structured scheme are needed. The E-HOD registry is collecting data on a large number of patients to create a large, standardized database and thereby facilitate and support considerations on whether to recommend inclusion or exclusion of these disorders into/from NBS panels.

Acknowledgments We gratefully acknowledge the support of Drs. Kimberley Chapman, Brian Fowler and Martin Lindner, and of Andrej Kutarn̆a, M.A.

The E-HOD project (No.2012_12_02) has received funding from the European Union in the framework of the Health Program. V.K. was in addition supported by research program RVO-VFN 64165/2012.

Compliance with Ethics Guidelines This article does not contain any studies with human or animal subjects performed by any of the authors.

Conflict of Interest None.

\section{References}

Abeling NG, van Gennip AH, Blom H, Wevers RA, Vreken P, van Tinteren HL, Bakker HD (1999) Rapid diagnosis and methionine administration: basis for a favourable outcome in a patient with methylene tetrahydrofolate reductase deficiency. J Inherit Metab Dis 22:240-242

Accinni R, Campolo J, Parolini M et al (2003) Newborn screening of homocystinuria: quantitative analysis of total homocys$\mathrm{t}(\mathrm{e})$ ine on dried blood spot by liquid chromatography with fluorimetric detection. J Chromatogr B Anal Technol Biomed Life Sci 785:219-226 
Adam S, Almeida SF, Weber EC (2013) Dietary practices in pyridoxine non-responsive homocystinuria: a European survey. Mol Gen Metab 110:454-459

Alfadhel M, Lillquist YP, Davis C, Junker AK, Stockler-Ipsiroglu S (2011) Eighteen-year follow-up of a patient with cobalamin F disease (cblF): report and review. Am J Med Genet 155:2571-2577

Alodaib AN, Carpenter K, Wiley V, Wotton T, Christodoulou J, Wilcken B (2012) Homocysteine measurement in dried blood spot for neonatal detection of homocystinurias. JIMD Rep 5:1-6

Armour CM, Brebner A, Watkins D, Geraghty MT, Chan A, Rosenblatt DS (2013) A patient with an inborn error of vitamin B12 metabolism (cblF) detected by newborn screening. Pediatrics 132:e257

Baric I (2009) Inherited disorders in the conversion of methionine to homocysteine. J Inherit Metab Dis 32:459-471

Barić I, Ćuk M, Fumić K, Vugrek O, Allen RH, Glenn B, Maradin M, Pažanin L, Pogribny I, Radoš M, Sarnavka V, Schulze A, Stabler S, Wagner C, Zeisel SH, Mudd SH (2005) S-Adenosylhomocysteine hydrolase deficiency: a second patient, the younger brother of the index patient, and outcomes during therapy. J Inherit Metab Dis 28: 885-902

Bártl J, Chrastina P, Krijt J, Hodík J, Pešková K, Kožich V (2014) Simultaneous determination of cystathionine, total homocysteine, and methionine in dried blood spots by liquid chromatography/ tandem mass spectrometry and its utility for the management of patients with homocystinuria. Clin Chim Acta 437:211-217

Bjursell MK, Blom HJ, Cayuela JA et al (2011) Adenosine kinase deficiency disrupts the methionine cycle and causes hypermethioninemia, encephalopathy, and abnormal liver function. Am J Hum Genet 89:507-515

Bowron A, Barton A, Scott J, Stansbie D (2005) Blood spot homocysteine: a feasibility and stability study. Clin Chem 51:257-258

Buist NR, Glenn B, Vugrek O, Wagner C, Stabler S, Allen RH, Pogribny I, Schulze A, Zeisel SH, Baric I, Mudd SH (2006) SAdenosylhomocysteine hydrolase deficiency in a 26-year-old man. $\mathrm{J}$ Inherit Metab Dis 29:538-545

Carrillo-Carrasco N, Chandler R, Venditti CP (2012) Combined methylmalonic acidemia and homocystinuria, cblC type. I. Clinical presentations, diagnosis and management. J Inherit Metab Dis 35: 91-102

Chace DH, Hillman SL, Millington DS, Kahler SG, Adam BW, Levy HL (1996) Rapid diagnosis of homocystinuria and other hypermethioninemias from newborns' blood spots by tandem mass spectrometry. Clin Chem 42:349-355

Coelho D, Kim JC, Miousse IR et al (2012) Mutations in ABCD4 cause a new inborn error of vitamin B12 metabolism. Nat Genet 44: $1152-1155$

Couce ML, Bóveda MD, García-Jimémez C et al (2013) Clinical and metabolic findings in patients with methionine adenosyltransferase I/III deficiency detected by newborn screening. Mol Genet Metab 110:218-221

Diekman EF, de Koning TJ, Verhoeven-Duif NM, Rovers MM, van Hasselt PM (2014) Survival and psychomotor development with early betaine treatment in patients with severe methylenetetrahydrofolate reductase deficiency. JAMA Neurol 71:188-194

Fattal-Valevski A, Bassan H, Korman SH, Lerman-Sagie T, Gutman A, Harel S (2000) Methylenetetrahydrofolatereductase deficiency: importance of early diagnosis. J Child Neurol 15:539-543

Fischer S, Huemer M, Baumgartner M et al (2014) Clinical presentation and outcome in a series of 88 patients with the cblC defect. J Inherit Metab Dis 37:831-840

Fowler B (1998) Genetic defects of folate and cobalamin metabolism. Eur J Pediatr 157(Suppl 2):S60-66

Fowler B, Schutgens RB, Rosenblatt DS, Smit GP, Lindemans J (1997) Folate-responsive homocystinuria and megaloblastic anaemia in a female patient with functional methionine synthase deficiency (cblE disease). J Inherit Metab Dis 20:731-741
Fu X, Xu YK, Chan P, Pattengale PK (2013) Simple, fast, and simultaneous detection of plasma total homocysteine, methylmalonic acid, methionine, and 2-methylcitric acid using liquid chromatography and mass spectrometry (LC/MS/MS). JIMD Rep 10:69-78

Gailus S, Suormala T, Malerczyk-Aktas AG et al (2010) A novel mutation in LMBRD1 causes the cblF defect of vitamin B(12) metabolism in a Turkish patient. J Inherit Metab Dis 33:17-24

Gan-Schreier H, Kebbewar M, Fang-Hoffmann J et al (2010) Newborn population screening for classic homocystinuria by determination of total homocysteine from Guthrie cards. J Pediatr 156:427-432

Gaustadnes M, Ingerslev J, Rütiger N (1999) Prevalence of congenital homocystinuria in Denmark. N Engl J Med 340:1513

Grubbs R, Vugrek O, Deisch J et al (2010) S-Adenosylhomocysteine hydrolase deficiency: Two siblings with fetal hydrops and fatal outcomes. J Inherit Metab Dis 33:705-713

Hall PL, Marquardt G, McHugh DM, Currier RJ, Tang H, Stoway SD, Rinaldo P (2014) Postanalytical tools improve performance of newborn screening by tandem mass spectrometry. Genet Med 16:889-895

Harding CO, Arnold G, Barness LA, Wolff JA, Rosenblatt DS (1997) Functional methionine synthase deficiency due to cblG disorder: a report of two patients and a review. Am J Med Genet 71:384-390

Honzík T, Magner M, Krijt J et al (2012) Clinical picture of Sadenosylhomocysteine hydrolase deficiency resembles phosphomannomutase 2 deficiency. Mol Genet Metab 107:611-613

Huemer M, Scholl-Bürgi S, Hadaya K et al (2014a) Three new cases of late-onset cblC defect and review of the literature illustrating when to consider inborn errors of metabolism beyond infancy. Orphanet J Rare Dis 9:161

Huemer M, Bürer C, Ješina P et al (2014b) Clinical onset and course, response to treatment and outcome in 24 patients with the cblE or cblG remethylation defect complemented by genetic and in vitro enzyme study data. J Inherit Metab Dis. doi:10.1007/s10545-0149803-7

Janosík M, Sokolová J, Janosíková B, Krijt J, Klatovská V, Kozich V (2009) Birth prevalence of homocystinuria in Central Europe: frequency and pathogenicity of mutation c.1105C > T (p.R369C) in the cystathionine beta-synthase gene. J Pediatr 154:431-437

Jusufi J, Suormala T, Burda P, Fowler B, Froese DS, Baumgartner MR (2014) Characterization of functional domains of the cblD (MMADHC) gene product. J Inherit Metab Dis 37: 841-849

Kim JC, Lee NC, Hwu PW et al (2012) Late onset of symptoms in an atypical patient with the cblJ inborn error of vitamin B12 metabolism: diagnosis and novel mutation revealed by exome sequencing. Mol Genet Metab 107:664-668

la Marca G, Malvagia S, Pasquini E, Innocenti M, Donati MA, Zammarchi E (2007) Rapid 2nd-tier test for measurement of 3$\mathrm{OH}$-propionic and methylmalonic acids on dried blood spots: reducing the false-positive rate for propionylcarnitine during expanded newborn screening by liquid chromatography-tandem mass spectrometry. Clin Chem 53:1364-1369

Lawrance AK, Racine J, Deng L, Wang X, Lachapelle P, Rozen R (2011) Complete deficiency of methylenetetrahydrofolate reductase in mice is associated with impaired retinal function and variable mortality, hematological profiles, and reproductive outcomes. J Inherit Metab Dis 34:147-157

Lerner-Ellis JP, Anastasio N, Liu J et al (2009) Spectrum of mutations in MMACHC, allelic expression, and evidence for genotypephenotype correlations. Hum Mutat 30:1072-1081

Magner M, Krupková L, Honzík T, Zeman J, Hyánek J, Kožich V (2011) Vascular presentation of cystathionine beta-synthase deficiency in adulthood. J Inherit Metab Dis 34:33-37

Marquardt G, Currier R, McHugh DM et al (2012) Enhanced interpretation of newborn screening results without analyte cutoff values. Genet Med 14:648-655 
Martinelli D, Deodato F, Dionisi-Vici C (2011) Cobalamin C defect: natural history, pathophysiology, and treatment. J Inherit Metab Dis 34:127-135

Martins E, Marcão A, Bandeira A, Fonseca H, Nogueira C, Vilarinho L (2012) Methionine adenosyltransferase I/III deficiency in Portugal: high frequency of a dominantly inherited form in a small area of douro high lands. JIMD Rep 6:107-112

McHugh D, Cameron CA, Abdenur JE et al (2011) Clinical validation of cutoff target ranges in newborn screening of metabolic disorders by tandem mass spectrometry: a worldwide collaborative project. Genet Med 13:230-54

Miousse IR, Watkins D, Coelho D et al (2009) Clinical and molecular heterogeneity in patients with the cblD inborn error of cobalamin metabolism. J Pediatr 154:551-556

Miousse IR, Watkins D, Rosenblatt DS (2011) Novel splice site mutations and a large deletion in three patients with the cblF inborn error of vitamin B12 metabolism. Mol Genet Metab 102:505-507

Mudd SH (2011) Hypermethioninemias of genetic and non-genetic origin: a review. Am J Med Genet C: Semin Med Genet 157C:3-32

Mudd SH, Skovby F, Levy HL et al (1985) The natural history of homocystinuria due to cystathionine beta-synthase deficiency. Am J Hum Genet 37:1-31

Müller P, Horneff G, Hennermann JB (2007) A rare inborn error of intracellular processing of cobalamin presenting with microcephalus and megaloblastic anemia: a report of 3 children. Klin Padiatr 219:361-367

Mulvihill A, Yap S, O'Keefe M, Howard PM, Naughten ER (2001) Ocular findings among patients with late-diagnosed or poorly controlled homocystinuria compared with a screened, well-controlled population. JAAPOS 5:311-315

Naughten ER, Yap S, Mayne PD (1998) Newborn screening for homocystinuria: Irish and world experience. Eur J Pediatr 157(Suppl 2):S84-87

Peterschmitt MJ, Simmons JR, Levy HL (1999) Reduction of false negative results in screening of newborns for homocystinuria. N Engl J Med 341:1572-1576

Refsum H, Fredriksen A, Meyer K, Ueland PM, Kase BF (2004) Birth prevalence of homocystinuria. J Pediatr 144:830-832

Rosenblatt DS, Aspler AL, Shevell MI, Pletcher BA, Fenton WA, Seashore MR (1997) Clinical heterogeneity and prognosis in combined methylmalonic aciduria and homocystinuria (cblC). J Inherit Metab Dis 20:528-538

Rutsch F, Gailus S, Miousse IR et al (2009) Identification of a putative lysosomal cobalamin exporter altered in the $\mathrm{cblF}$ defect of vitamin B12 metabolism. Nat Genet 41:234-239

Schiff M, Benoist JF, Tilea B, Royer N, Giraudier S, Ogier de Baulny H (2011) Isolated remethylation disorders: do our treatments benefit patients? J Inherit Metab Dis 34:137-145

Scolamiero E, Villani GR, Ingenito L et al (2014) Maternal vitamin B12 deficiency detected in expanded newborn screening. Clin Biochem 47:312-317

Shih VE, Axel SM, Tewksbury JC, Watkins D, Cooper BA, Rosenblatt DS (1989) Defective lysosomal release of vitamin B12 (cb1F): a hereditary cobalamin metabolic disorder associated with sudden death. Am J Med Genet 33:555-563

Skovby F, Gaustadnes M, Mudd SH (2010) A revisit to the natural history of homocystinuria due to cystathionine beta-synthase deficiency. Mol Genet Metab 99:1-3
Stabler SP, Korson M, Jethva R et al (2013) Metabolic profiling of total homocysteine and related compounds in hyperhomocysteinemia: utility and limitations in diagnosing the cause of puzzling thrombophilia in a family. JIMD Rep 11:149-163

Suormala T, Baumgartner MR, Coelho D et al (2004) The cblD defect causes either isolated or combined deficiency of methylcobalamin and adenosylcobalamin synthesis. J Biol Chem 279:42742-42749

Surtees R, Leonard J, Austin S (1991) Association of demyelination with deficiency of cerebrospinal-fluid S-adenosylmethionine in inborn errors of methyl-transfer pathway. Lancet 338:1550-1554

Thomas MA, Rosenblatt DS (2005) Severe methylenetetrahydrofolate reductase deficiency. In: Ueland PM \& Rozen R, editors. MTHFR polymorphisms and disease. Eureka; p.41-53.

Tortorelli S, Turgeon CT, Lim JS et al (2010) Two-tier approach to the newborn screening of methylenetetrahydrofolate reductase deficiency and other remethylation disorders with tandem mass spectrometry. J Pediatr 157:271-275

Turgeon CT, Magera MJ, Cuthbert CD et al (2010) Determination of total homocysteine, methylmalonic acid, and 2-methylcitric acid in dried blood spots by tandem mass spectrometry. Clin Chem 56: 1686-1695

Vilaseca MA, Vilarinho L, Zavadakova P et al (2003) CblE type of homocystinuria: mild clinical phenotype in two patients homozygous for a novel mutation in the MTRR gene. J Inherit Metab Dis 26:361-369

Walter JH, Wraith JE, White FJ, Bridge C, Till J (1998) Strategies for the treatment of cystathionine beta-synthase deficiency: the experience of the willink biochemical genetics unit over the past 30 years. Eur J Pediatr 57(Suppl 2):S71-76

Watkins D, Rosenblatt DS (2012) Update and new concepts in vitamin responsive disorders of folate transport and metabolism. J Inherit Metab Dis 35:665-670

Weisfeld-Adams JD, Morrissey MA, Kirmse BM et al (2010) Newborn screening and early biochemical follow-up in combined methylmalonic aciduria and homocystinuria, cblC type, and utility of methionine as a secondary screening analyte. Mol Genet Metab 99:116-123

Weisfeld-Adams JD, Bender HA, Miley-Åkerstedt A et al (2013) Neurologic and neurodevelopmental phenotypes in young children with early-treated combined methylmalonic acidemia and homocystinuria, cobalamin C type. Mol Genet Metab 110:241-247

Wilson JM, Jungner YG (1968) Principles and practice of mass screening for disease. Public Health Pap World Health Organ 34:26-39

Yap S, Naughten E (1998) Homocystinuria due to cystathionine bsynthase deficiency in Ireland: 25 years experience of a newborn screened and treated population with reference to clinical outcome and biochemical control. J Inherit Metab Dis 21:738-747

Yap S, Boers GH, Wilcken B et al (2001) Vascular outcome in patients with homocystinuria due to cystathionine beta-synthase deficiency treated chronically: a multicenter observational study. Arterioscler Thromb Vasc Biol 21:2080-2085

Zavadakova P, Fowler B, Suormala T et al (2005) cblE Type of Homocystinuria due to methionine synthase reductase deficiency: functional correction by minigene expression. Hum Mutat 25: 239-247 